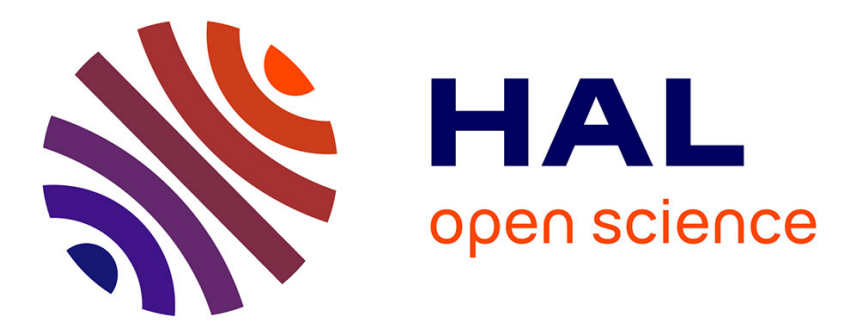

\title{
Observations of the relationship between frequency sweep rates of chorus wave packets and plasma density
}

E Macúšová, O Santolík, Pierrette Décréau, A. G. Demekhov, D Nunn, D.A. Gurnett, J.S. Pickett, E. E. Titova, B. V. Kozelov, Jean-Louis Rauch, et al.

\section{- To cite this version:}

E Macúšová, O Santolík, Pierrette Décréau, A. G. Demekhov, D Nunn, et al.. Observations of the relationship between frequency sweep rates of chorus wave packets and plasma density. Journal of Geophysical Research Space Physics, 2010, 115, A12257 (8 p.). 10.1029/2010JA015468 . insu01180755

\section{HAL Id: insu-01180755}

https://hal-insu.archives-ouvertes.fr/insu-01180755

Submitted on 28 Jul 2015

HAL is a multi-disciplinary open access archive for the deposit and dissemination of scientific research documents, whether they are published or not. The documents may come from teaching and research institutions in France or abroad, or from public or private research centers.
L'archive ouverte pluridisciplinaire HAL, est destinée au dépôt et à la diffusion de documents scientifiques de niveau recherche, publiés ou non, émanant des établissements d'enseignement et de recherche français ou étrangers, des laboratoires publics ou privés. 


\title{
Observations of the relationship between frequency sweep rates of chorus wave packets and plasma density
}

\author{
E. Macúšová, ${ }^{1,2}$ O. Santolík, ${ }^{1,2}$ P. Décréau, ${ }^{3}$ A. G. Demekhov, ${ }^{4}$ D. Nunn, ${ }^{5}$ D. A. Gurnett, ${ }^{6}$ \\ J. S. Pickett, ${ }^{6}$ E. E. Titova, ${ }^{7,8}$ B. V. Kozelov, ${ }^{7}$ J.-L. Rauch, ${ }^{3}$ and J.-G. Trotignon ${ }^{3}$ \\ Received 16 March 2010; revised 3 September 2010; accepted 5 October 2010; published 31 December 2010.
}

[1] Chorus emissions are generated by a nonlinear mechanism involving wave-particle interactions with energetic electrons. Discrete chorus wave packets are narrowband tones usually rising (sometimes falling) in frequency. We investigate frequency sweep rates of chorus wave packets measured by the Wideband data (WBD) instrument onboard the Cluster spacecraft. In particular, we study the relationship between the sweep rates and the plasma density measured by the WHISPER active sounder. We have observed increasing values of the sweep rate for decreasing plasma densities. We have compared our results with results of simulations of triggered emissions as well as with estimates based on the backward wave oscillator model for chorus emissions. We demonstrate a reasonable agreement of our experimental results with theoretical ones.

Citation: Macúšová, E., et al. (2010), Observations of the relationship between frequency sweep rates of chorus wave packets and plasma density, J. Geophys. Res., 115, A12257, doi:10.1029/2010JA015468.

\section{Introduction}

[2] Whistler mode chorus is a common type of very low frequency (VLF) emission observed in the Earth's magnetosphere [Gurnett and O'Brien, 1964] and in other planetary magnetospheres [Gurnett et al., 1981; Menietti et al., 2008; Hospodarsky et al., 2008] and detected on the ground [Storey, 1953; Manninen, 2005; Gotkowski and Inan, 2008]. Whistler mode chorus emissions are generated by a nonlinear mechanism through a cyclotron instability with anisotropic distributions of energetic electrons (with energies between a few $\mathrm{keV}$ and several tens of $\mathrm{keV}$ [Trakhengerts, 1963; Kennel and Petschek, 1966; Tsurutani and Smith, 1974]) in the Earth's magnetic field, when discrete narrowband wave packets (chorus elements) of short duration

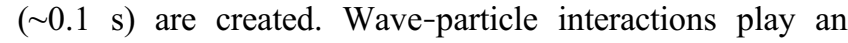
important role in the acceleration of electrons in the radiation belts to relativistic energies as well as in the losses of energetic electrons during geomagnetic storms [Horne and Thorne, 1998, 2003; Horne et al., 2003, 2005; Meredith

\footnotetext{
${ }^{1}$ Faculty of Mathematics and Physics, Charles University, Prague, Czech Republic.

${ }^{2}$ Institute of Atmospheric Physics, Academy of Sciences of the Czech Republic, Prague, Czech Republic.

${ }^{3}$ Laboratoire de Physique et Chimie de l'Environnement et de l'Espace, CNRS, University of Orléans, Orléans, France.

${ }^{4}$ Institute of Applied Physics, Russian Academy of Sciences, Nizhny Novgorod, Russia.

${ }^{5}$ ECS, Southampton University, Southampton, UK.

${ }^{6}$ Department of Physics and Astronomy, University of Iowa, Iowa City, Iowa, USA.

${ }^{7}$ Polar Geophysical Institute, Apatity, Russia.

${ }^{8}$ Space Research Institute, Russian Academy of Sciences, Moscow, Russia.

Copyright 2010 by the American Geophysical Union. 0148-0227/10/2010JA015468
}

et al., 2002, 2003; Santolik et al., 2004a; Demekhov et al., 2006].

[3] Spacecraft and ground based observations help to characterize the source of chorus emissions, which is located within a few degrees of the magnetic equator and its characteristic scale parallel to the magnetic field $\mathbf{B}_{\mathbf{0}}$ lies in the range 3000-5000 km [LeDocq et al., 1998; Santolik et al., 2004a] while the characteristic size perpendicular to $\mathbf{B}_{\mathbf{0}}$ is between 60 and $200 \mathrm{~km}$ [Santolik et al., 2004b]. Near the source region chorus emissions occur in two distinct frequency bands (banded chorus) lying at frequencies from a few hundred $\mathrm{Hz}$ to several $\mathrm{kHz}$ with a gap at one half of the equatorial electron cyclotron frequency $\left(f_{c e}\right)$. Banded chorus was positively correlated with magnetic activity [Anderson and Maeda, 1977]. Suggestion, that Landau damping is one of the possible mechanisms explaining the existence of the gap was first mentioned by Tsurutani and Smith [1974]. Omura et al. [2009] solved this problem by a system of equations based on the nonlinear Landau damping. In recent studies Bell et al. [2009] discuss the role of ducts in formation of the gap. The frequency range of the lower band extends from 0.1 to $0.5 f_{c e}$ and the upper band extends from 0.5 to $0.7 f_{c e}$ [Burtis and Helliwell, 1969; Tsurutani and Smith, 1977; Meredith et al., 2001]. The majority of chorus wave packets are rising in frequency (risers) from a few $\mathrm{kHz}$ to a few tens of $\mathrm{kHz}$ per second; falling tones (fallers) are also observed [Sazhin and Hayakawa, 1992].

[4] Since the spectral forms of the chorus elements and of triggered ELF/VLF emissions [Nunn et al., 1997] are very similar, the theory of triggered signals can be used as a starting point for understanding chorus generation. The basic parameters of chorus elements can also be estimated on the basis of the backward wave oscillator (BWO) model [Trakhtengerts, 1995, 1999; Trakhtengerts et al., 2004] and on the basis of the theories proposed by Omura et al. [2008, 
2009]. For example, the BWO model predicts that the chorus frequency sweep rate $d f / d t$ is proportional on the wave amplitude $B_{w}$. This is based on the suggestion that the frequency drift results from sequential generation of sidebands. Similar dependence of $d f / d t$ on $B_{w}$ has also been obtained by Omura et al. [2008] using another approach, namely, by analyzing the maximum nonlinear growth rate under an assumption that the electron distribution has a plateau within the nonlinear trapping region of the phase space.

[5] The aim of this paper is to present an experimental study of chorus frequency sweep rates $d f / d t$. Since the experimental analysis of wave amplitudes $B_{w}$ is a complex problem, we first aim at analysis of $d f / d t$ as a function of the background plasma density which has been noted in numerical simulations by Nunn et al. [2005]. The analysis of the dependence of $d f / d t$ on the wave amplitude $B_{w}$ is beyond the scope of this paper and results will be reported separately when they become available.

[6] In the present paper, we analyze several chorus events with significantly different plasma densities and we compare the results with a theoretical model and numerical simulations. We analyze the statistical distribution of $d f / d t$ for these events and we obtain a relationship between the median frequency sweep rate of chorus wave packets and the median plasma density, which can be compared with theoretical predictions. For this comparison, we use the backward wave oscillator model and numerical simulations of triggered emissions, since both these models yield similar scalings of $d f / d t$ with $n_{e}$, which can be separated from the dependencies on other parameters. Section 2 explains this expected scaling of $d f / d t$ as a function of the plasma density based on these models. Selection criteria for chorus emissions used in this analysis are defined in section 3 . The analyzed data set is briefly described in section 4. This section also shows an example of chorus wave packets, and examples of histograms of the frequency sweep rates. Section 5 contains results of our experimental study and a comparison of observations with the theoretical scaling based on the model of Trakhtengerts et al. [2004] and with the numerical simulations of Nunn et al. [2005, 2009]. Section 6 presents brief conclusions.

\section{Theoretical Background}

[7] Nunn [1990] developed the VHS (Vlasov Hybrid Simulation) technique, applied it to the problem of nonlinear wave-particle interactions in the VLF band, and used it for numerical simulations of the triggered emissions. The code produces stable reproducible emissions whose characteristics agree with experimental observations. Both triggered and chorus VLF emissions are formed via nonlinear cyclotron wave-particle interactions, and an individual element can be regarded as an emission triggered by the previous element. The physics of the nonlinear frequency shift in chorus and triggered emissions seems to be basically the same. Numerical simulations based on the 1-D VHS code show the physics of the triggering process and also how the properties of the triggered emissions depend upon different parameters.

[8] Nunn et al. [2005] systematically varied each input parameter to investigate how the triggered emissions depend on the individual simulation parameters and what factor determines the emission frequency sweep rate. The input parameters were as follows: the linear growth rate, the magnitude of the input wave pulse, the plasma density, the triggering pulse length, the saturation amplitude employed in the code, and the simulation bandwidth. It was found that the sweep rate depended most strongly on the plasma density, with the sweep rate magnitude decreasing as the density increased.

[9] Trakhtengerts [1995, 1999] suggested a generation mechanism of chorus emissions based on the BWO (Backward Wave Oscillator) regime of wave-particle interactions and a nonlinear wave growth. When a step-like deformation in a distribution function of energetic electrons in the parallel velocity component with respect to the magnetic field is present, an absolute instability of whistler mode waves can occur. If a sharp enough gradient in parallel velocities is absent, the linear phase of this instability requires very high fluxes and anisotropies of energetic electrons at lower equatorial radial distances of about 4-5 Earth radii. Note that at higher radial distances, in agreement with the results of $\mathrm{Li}$ et al. [2010], one-hop gain can be high enough to develop the instability in another regime, but our data are obtained at lower radial distances. That is the main reason, why we assume the step-like feature. This deformation can come from the cyclotron interactions of electrons with noise-like emissions. The electrons having a step-like distribution and moving along the magnetic field lines generate chorus emissions propagating in the opposite direction.

[10] The BWO theory provides us with quantitative estimates for basic parameters of the chorus emissions, such as the chorus growth rate, the wave amplitude and the frequency sweep rate. The growth rate of the absolute (BWO) instability is described by Trakhtengerts et al. [2004, equation (4)]. The growth rate determined by this equation and the trapping frequency determined by Trakhtengerts et al. [2004, equation (14)] allow us to estimate the characteristic chorus wave amplitude in a different way. These two parameters are related by Trakhtengerts et al. [2004, equation (13)]. From Trakhtengerts et al. [2004, equations (13) and (14)] we can calculate the value of the wave amplitude. In this theory, the frequency of the chorus emissions is determined by the parallel velocity of the electrons, corresponding to a step-like deformation in the distribution function of resonant electrons [Trakhtengerts et al., 2007].

[11] To compare the observations with the theoretical estimates based on the BWO model and numerical simulations, we calculate the median values of the sweep rates $(d f / d t)$ for each event. We then use a rough theoretical estimate of the frequency sweep rate given by Trakhtengerts et al. [2004, equation (16)], according to which

$$
\frac{d f}{d t} \approx 0.5 \gamma_{B W O}^{2}
$$

where $\gamma_{B W O}$ is the growth rate of the absolute instability at the linear stage of the whistler mode wave growth. When we take into account also Trakhtengerts et al. [2004, equation (13)], we can note that these two formulas for the sweep rate are actually similar to that given by Omura et al. [2008, equation (49)], who use a different description of the mechanism of the frequency sweeping. 
[12] Equation (1) combines the proportional scaling of $d f / d t$ with wave amplitude [Trakhtengerts, 1999; Omura et al., 2008] and an estimate for the wave saturation amplitude based on the nonlinear theory of the cyclotron instability [see Trakhtengerts et al., 2004]. Such a combination allows us to exclude the wave amplitude from the formula for $d f / d t$. The growth rate can be then estimated according to a corrected version of equation (4) of Trakhtengerts et al. [2004]:

$$
\gamma_{B W O}=\frac{\pi^{2}}{4 T_{0}} Q(Q-1)
$$

[13] Here, $Q$ is the dimensionless parameter proportional to the square root of the density of energetic electrons related to the threshold for the BWO regime. According to a newer analysis by Bespalov and Demekhov [2009], equation (2) is valid for $Q \approx 1$, and for $Q>1$, another equation can be used:

$$
\gamma_{B W O} \approx \frac{\pi}{2 T_{0}}\left(Q-Q^{-2 / 3}\right)
$$

$T_{0}$ in equations (2) and (3) is the characteristic time scale of the BWO:

$$
T_{0}=l_{B W O}\left(\frac{1}{v_{*}}+\frac{1}{v_{g}}\right)
$$

where $l_{B W O}$ is the characteristic length of the BWO system (chorus source region), and $v_{*}$ and $v_{g}$ are the absolute values of the parallel velocity of resonant electrons and the group velocity of the whistler mode waves, respectively.

[14] An estimate for $l_{B W O}$ is given by Trakhtengerts et al. [2004, equation (3)]:

$$
l_{B W O} \approx 1.76\left(\frac{2 \pi R_{E}^{2} L^{2}}{6 k}\right)^{1 / 3}
$$

where $L$ is the McIlwain parameter, $R_{E}$ is the Earth's radius, and $k$ if the wave number.

[15] Assuming $f_{p}^{2} \gg f\left(f-f_{c e}\right)$, where $f_{p}$ is the plasma frequency, and $f_{c e}$ is the electron cyclotron frequency, and assuming further propagation with wave vectors parallel to the background magnetic field $\mathbf{B}_{\mathbf{0}}$, we can use a simplified cold plasma whistler mode dispersion relation:

$$
f=\frac{f_{c e} k^{2} c^{2}}{f_{p}^{2}+k^{2} c^{2}}
$$

This relation can be used to estimate the group velocity $v_{g}$ :

$$
v_{g}=2 \pi \frac{\partial f}{\partial k}=\frac{4 \pi F\left(f_{c e}-f\right)}{k f_{c e}}
$$

The condition of the first-order cyclotron resonance gives us the resonant parallel velocity $v_{*}$ :

$$
v_{*}=2 \pi \frac{f_{c e}-f}{k}
$$

[16] Combining equations (1), (3), (4), (5), (7), and (8) with the relation of the plasma frequency $f_{p}$ to the plasma density, $f_{p}^{2}=n_{e} e^{2} m_{e}^{-1} \epsilon_{0}^{-1}$, (where $e, m_{e}$, and $\epsilon_{0}$, respectively, are the electron charge, mass, and the vacuum permittivity), and separating the dependence on the plasma density $n_{e}$, we obtain the following scaling:

$$
\frac{d f}{d t} \approx a n_{e}^{-2 / 3}
$$

where

$$
a \approx 240 \frac{\left(Q-Q^{-2 / 3}\right)^{2} c^{4 / 3} m_{e}^{2 / 3} \epsilon_{0}^{2 / 3}}{R_{E}^{4 / 3} L^{4 / 3} e^{4 / 3}} \frac{\left(f_{c e}-f\right)^{8 / 3} f^{4 / 3}}{\left(2 f+f_{c e}\right)^{2}}
$$

[17] For simplicity, we neglect the fact that $a$ depends on $f$ and $f_{c e}$ in our analysis. Furthermore, $L$ values of our observations are close to each other and approximately equal to 4.4. The most significant unknown in this scaling is the parameter $Q$ which quantifies the excess of energetic electron density above the generation threshold. The simulations of Demekhov and Trakhtengerts [2008] show that chorus elements are formed only if $Q$ is sufficiently high, and that they are not formed if $Q$ is only marginally above unity. This is also the reason why we use equation (3) (for $Q>1$ ) instead of equation (2) (for $Q \approx 1$ ) in evaluating an estimate of the scaling factor $a$ in equation (10). To make the comparison with the observations possible, we, however, have to impose a hypothesis that the parameter $Q$ is on average independent of $n_{e}$ and that it is the same for all chorus events.

[18] We then use the relationship described by equation (9) to fit the experimental data, where we use the experimentally determined median value of the plasma density for $n_{e}, d f / d t$ is the median value of the observed frequency sweep rate, and $a$ is a free parameter. The value of $a$ is determined from a least squares fit of the model described by equation (9). To generalize our results, and to verify is the theoretical scaling from equation (9) well corresponds to our observations, we replace the exponent of $-2 / 3$ by another free parameter $b$ :

$$
\frac{d f}{d t}=a n_{e}^{-b}
$$

A linear fit of a logarithm of this equation gives an estimate of the values of the parameters $a$ and $b$.

\section{Analysis of the Chorus Sweep Rate}

[19] To compare the observations with the theoretical estimates based on the BWO model and numerical simulations, we have calculated the median values of the sweep rates $(d f / d t)$ for each event.

[20] We have analyzed measurements of the WBD instrument [Gurnett et al., 2001], the STAFF-SA instrument [Cornilleau-Wehrlin et al., 1997], and the WHISPER active sounder [Décréau et al., 2001] on board the four Cluster spacecraft. The WBD instrument provides us with highresolution measurements of one electric or one magnetic component. We use the data, which were measured in the instrument's lowest frequency band with a lower frequency of approximately $100 \mathrm{~Hz}$ and with a total bandwidth of $9.5 \mathrm{kHz}$. Three components of the wave magnetic field are measured by the STAFF-SA instrument, which has an upper frequency limit of $4 \mathrm{kHz}$. We calculate the parallel com- 


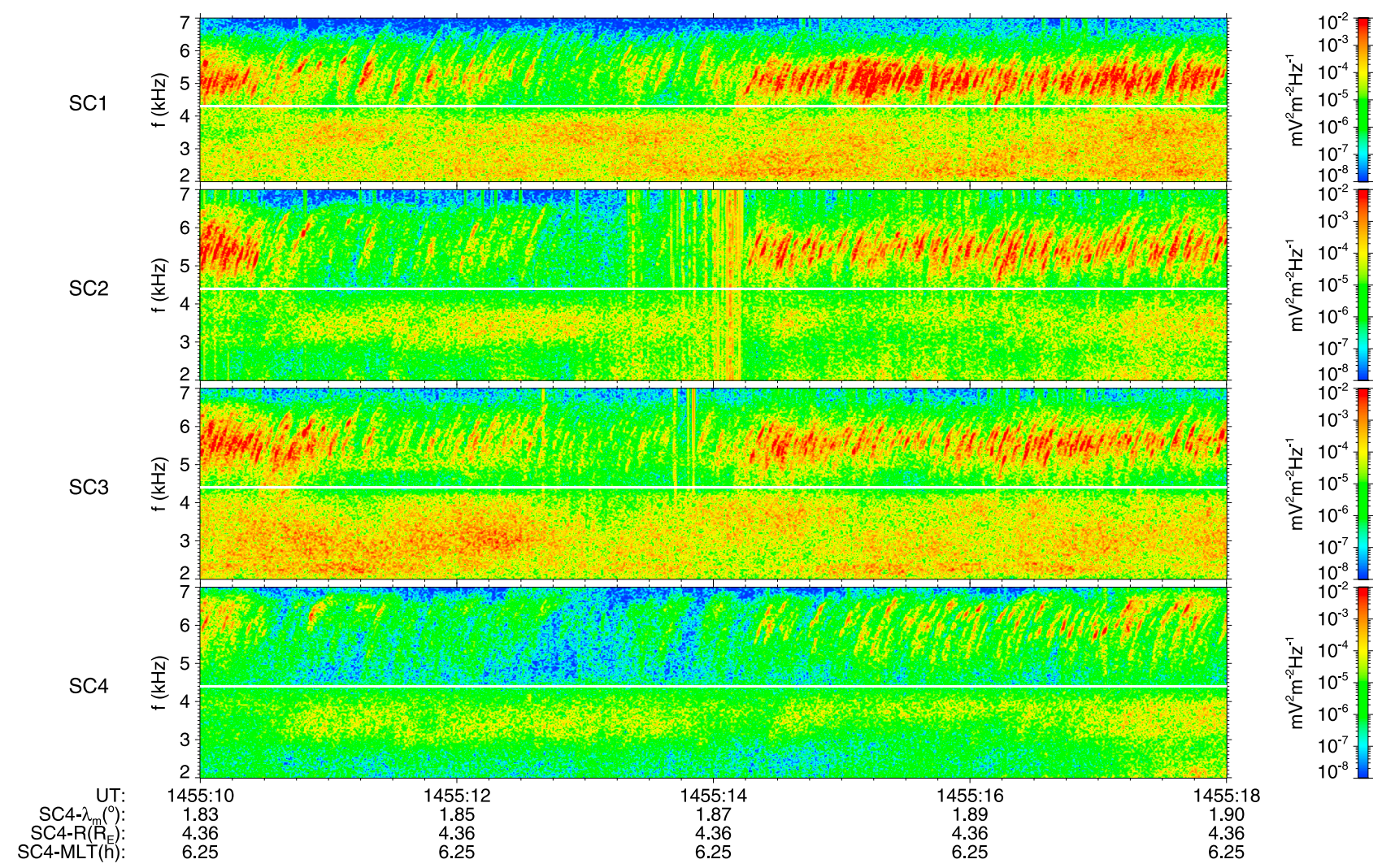

Figure 1. Example of chorus wave packets measured at $\mathrm{L}=4.2$ on 6 December 2003 by the Cluster satellites 1, 2, 3, and 4. Time and spacecraft position are given on the bottom: UT, universal time; $\lambda_{m}$, magnetic dipole latitude; $R_{E}$, Earth radius; MLT, magnetic local time. One half of the electron cyclotron frequency (white line) for satellite 1 is $4.3 \mathrm{kHz}$, for satellite 2 it is $4.4 \mathrm{kHz}$, for satellite 3 it is $4.3 \mathrm{kHz}$, and for satellite 4 it is $4.4 \mathrm{kHz}$.

ponent of the Poynting flux normalized by its standard deviation from the components of the magnetic and electric fields. This value, as well as the electromagnetic planarity parameter [Santolik et al., 2003], allows us to determine the localization and size of the source region of chorus emissions for each analyzed event using the same procedure as Santolik et al. [2004b]. The measurements from this region give us credible information about the frequency sweep rate in the generation region, because outside the source region the wave dispersion can change the inclination of the chorus wave packets in the frequency-time plane and therefore it has an influence on the value of the frequency sweep rate.

[21] This study is limited to intense chorus events where the power spectral density of electric field fluctuations (PSD) is larger than $10^{-5} \mathrm{mV}^{2} \mathrm{~m}^{-2} \mathrm{~Hz}^{-1}$. In addition to this criterion, individual chorus wave packets are required to be at least 2 orders of magnitude above the background. Data from the WBD instrument must be available for the identification of these chorus wave packets. To specify the total plasma density during the processed time intervals, data from the Whisper active sounder must also be available. From the beginning of the spacecraft mission in 2001 until the end of 2005, we found thirteen cases fulfilling the above mentioned criteria, which allow us to obtain the value of the frequency sweep rate for many thousands of wave packets. First, we have visually identified each chorus element (see Figure 1 for an example) on the frequency-time spectro- grams and then we have visually determined its starting frequency $f_{1}$, its ending frequency $f_{2}$, as well as the times $t_{1}$ and $t_{2}$ corresponding to its starting and ending frequency, respectively. In this study we do not take into account the hook shapes of the chorus elements; we assume that individual wave packets are risers linearly increasing their frequency, or fallers linearly decreasing their frequency as a function of time.

[22] From these values we have calculated the frequency sweep rate for each chorus wave packet as follows:

$$
\frac{d f}{d t}=\frac{f_{2}-f_{1}}{t_{2}-t_{1}}
$$

The first results of this method have been presented by Santolik et al. [2008].

\section{Data Set}

[23] All processed time intervals with different levels of geomagnetic activity, different median plasma densities and different $\mathrm{L}$ shells are listed in Table 1 . In all cases the source region was found near the geomagnetic equator. The events are listed in Table 1 in order of increasing density (obtained from the WHISPER instrument). The number of risers (increasing tones with the sweep rate $\frac{d f}{d t}>0$ ) and fallers (decreasing tones with the sweep rate $\frac{d f}{d t}<0$ ) and their 
Table 1. Median Values of Frequency Sweep Rate From All Processed Cases ${ }^{\mathrm{a}}$

\begin{tabular}{|c|c|c|c|c|c|c|c|c|c|}
\hline Date & Time (UT) & $L$ & $M L T$ & $n_{e}\left(\mathrm{~cm}^{-3}\right)$ & $K_{p}$ & $\left(\frac{d f}{d t}\right)_{R}(\mathrm{kHz} / \mathrm{s})$ & $N_{R}$ & $\left(\frac{d f}{d t}\right)_{F}(\mathrm{kHz} / \mathrm{s})$ & $N_{F}$ \\
\hline 18 Apr. 2002 & 08:38-09:05 & 4.4 & 21 & $4_{-1.4}^{+1.5}$ & $3^{+}$ & $7.54_{-2.3}^{+4.2}$ & 4678 & - & - \\
\hline 6 Dec. 2003 & $14: 30-15: 00$ & 4.6 & 6.4 & $10_{-0.6}^{+0.7}$ & $3^{0}$ & $9.36_{-3.4}^{+8.8}$ & 5945 & - & - \\
\hline 22 Dec. 2001 & $20: 10-20: 30$ & 4.2 & 5 & $10_{-2.6}^{+2.2}$ & $2^{0}$ & - & - & $-11.02_{-15.4}^{+4.4}$ & 953 \\
\hline 20 Jan. 2004 & $19: 25-19: 29$ & 4.3 & 2.8 & $11_{-0.2}^{+1.2}$ & $4^{+}$ & - & - & $-9.00_{-10.3}^{+3.7}$ & 417 \\
\hline 6 Jan. 2004 & $12: 30-12: 45$ & 4.4 & 4.2 & $12_{-0.6}^{+0.3}$ & $2^{+}$ & - & - & $-8.27_{-1,16}^{+4.1}$ & 952 \\
\hline 19 Nov. 2001 & $12: 00-12: 36$ & 4.5 & 7.4 & $12_{-4}^{+4}$ & $3^{+}$ & - & - & $-7.55_{-17.8}^{+4.5}$ & 2950 \\
\hline 12 Apr. 2001 & $04: 35-04: 57$ & 4 & 21.4 & $13_{-2.6}^{+2.2}$ & $6^{+}$ & $5.95_{-3}^{+15.2}$ & 365 & $-8.60_{-11.2}^{+4.4}$ & 173 \\
\hline 8 Feb. 2005 & $11: 50-12: 13$ & 4.2 & 1.8 & $16_{-0.7}^{+0.8}$ & $3^{+}$ & $10.45_{-5.6}^{+18.3}$ & 396 & $-13.81_{-20.1}^{+8.2}$ & 258 \\
\hline 6 Feb. 2004 & $10: 40-11: 08$ & 4.3 & 2 & $17_{-0.9}^{+0.2}$ & $4^{+}$ & $10.75_{-5.5}^{+10.7}$ & 2180 & - & - \\
\hline 13 Sep. 2001 & $20: 30-21: 30$ & 4.3 & 11.5 & $20_{-6.9}^{+1.6}$ & $2^{+}$ & - & - & $-3.92_{-4.8}^{+1.6}$ & 825 \\
\hline 25 Mar. 2002 & $13: 56-14: 21$ & 4.4 & 22.5 & $35_{-1.1}^{+1.9}$ & $2^{0}$ & $3.41_{-0.7}^{+2.9}$ & 431 & - & - \\
\hline 8 Dec. 2001 & $13: 10-13: 35$ & 4.2 & 6 & $41_{-11}^{+9.7}$ & $1^{-}$ & $3.38_{-1.2}^{+5.3}$ & 145 & - & - \\
\hline 21 Oct. 2001 & $23: 15-23: 35$ & 4.1 & 8.9 & $105_{-38}^{+75}$ & $8^{-}$ & $1.69_{-0.6}^{+1.7}$ & 988 & $-3.11_{-1.4}^{+1.5}$ & 851 \\
\hline
\end{tabular}

${ }^{\mathrm{a}}$ The following information is provided, by column number: 1 , date; 2 , time interval; 3, L; 4, MLT; 5 , median plasma density $n_{e-(\text { median }-P 14 / 100)}^{+(P 84 / 100-\text { median })}$; $6, K_{p}$ index; 7, median value of the frequency sweep rate $\left(\frac{d f}{d t}\right)_{R}$ for risers ${ }_{-(\text {median }-P 14 / 100)}^{+(P 84110-\text { median }} ; 8$, number of processed risers $N_{R} ; 9$, median value of $\left(\frac{d f}{d t}\right)_{F}$ for fallers ${ }_{-(\text {median }-P 14 / 100)}^{+(P 84 / 10-\text { median })}$ 10, number of processed falling tones $N_{F}$.

respective frequency sweep rates are provided separately. In most cases different spacecraft are passing through the source region in different times, which causes a time shift between starting and ending times of individual time subintervals from the different spacecraft. The first two columns in Table 1 therefore show the maximum time interval, enveloping all subintervals from the individual spacecraft.

[24] Both rising and falling tones were observed in three of the processed cases. The plasma density remains almost constant in two of them. For example, on 12 April 2001 we observed most of the risers during the entire time period in the upper band and most of fallers in the lower band. However, in this case the amplitude of chorus wave packets was amplified during their propagation away from the equator and this effect may have an influence on the variability of the value of $d f / d t$. A study based on a larger data set could manifest that also other factors can play important role in the variability of the $d f / d t$ during the analyzed time interval.

[25] Below are briefly described other three cases with examples of the probability density function of the frequency sweep rate.

[26] On 6 December 2003 from 14:30 to 15:00 UT at L = 4.6 and $\mathrm{MLT}=6.4$, mostly rising tones were observed (see

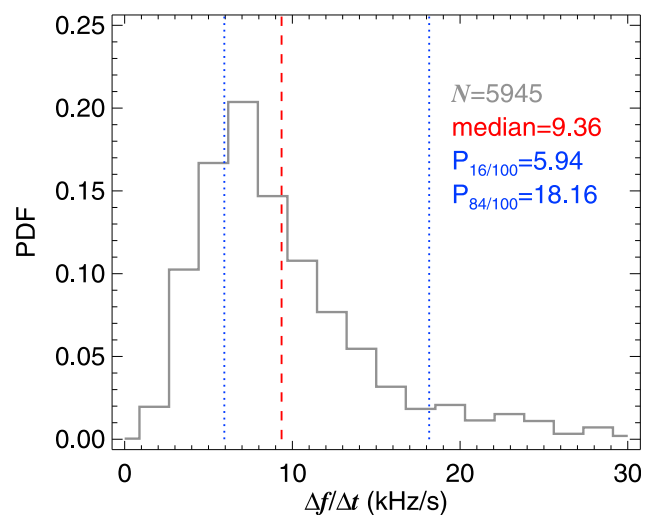

Figure 2. Probability density function (PDF) of the sweep rate of risers measured on 6 December 2003. The dashed line shows the median sweep rate; the dotted lines give the $P_{\frac{16}{100}}$ and $P_{\frac{84}{100}}$ percentiles. an 18 second example Figure 1). A histogram of the probability density function of the frequency sweep rates of these risers is given in Figure 2. In Figure 2 the median value of the sweep rate is marked by a red dashed line and two percentiles $P_{\frac{16}{16}}$ and $P_{\frac{84}{10}}$, corresponding to values separated by \pm one standard deviation from the mean value in an ideal case of a normal distribution, are marked by blue dotted lines. In our case these percentiles characterize the width and asymmetry of the probability density functions.

[27] Figure 3 provides a histogram of the frequency sweep rates on 19 November 2001 from 12:00 to 12:45 UT at L value 4.5 and MLT 7.4 when mostly fallers were observed.

[28] On 21 October 2001 from 23:15 to 23:35 UT, when the median plasma density was high at $105 \mathrm{~cm}^{-3}$ and the geomagnetic activity was very high at $\mathrm{L}=4.1$ and $\mathrm{MLT}=$ 8.9 both risers and fallers were observed. The histogram of the probability density functions of the sweep rates of risers for this event is given in Figure 4.

\section{Results of the Sweep Rate Study}

[29] During the thirteen time intervals with different values of the plasma density, we have observed chorus wave packets with different frequency drift rates in the frequencytime plane and with different extents in frequency and time.

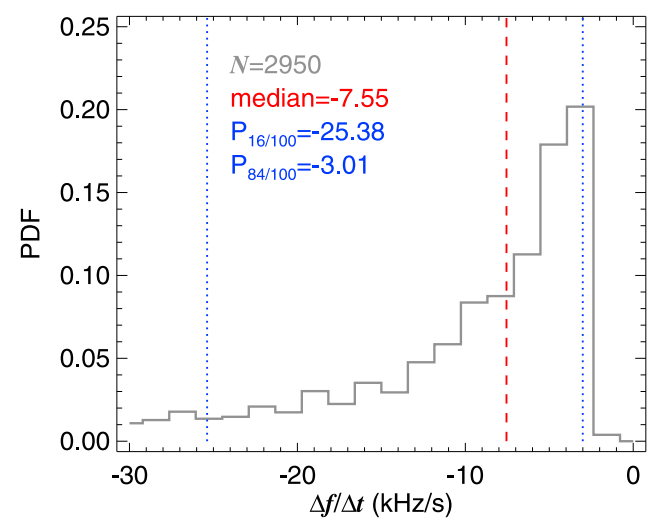

Figure 3. PDF of the sweep rate of fallers from 19 November 2001. The dashed line represents the median sweep rate; the dotted lines give the $P_{\frac{16}{100}}$ and $P_{\frac{84}{100}}$ percentiles. 


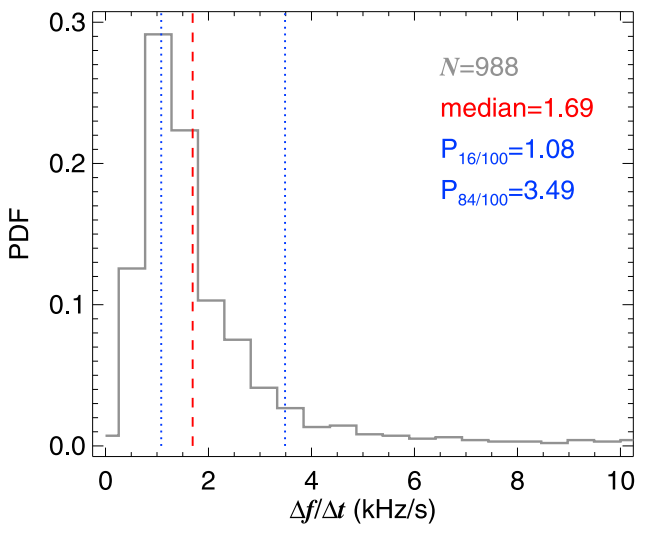

Figure 4. PDF of the sweep rate of risers from 21 October 2001. The dashed line labels the median sweep rate; the dotted lines describe the $P_{\frac{16}{100}}$ and $P_{\frac{84}{100}}$ percentiles.

The duration of individual chorus wave packets varied between 30 and $500 \mathrm{~ms}$; see an example for 6 December 2003 in Figure 5.

[30] Table 1 shows small affect of parameters MLT, $K_{p}$ and $L$ on the value of $d f / d t$, beyond plasma density, which is the most significant parameter for sweep rate. We cannot exclude, however, that a larger sample of processed cases might show some influence of the other parameters on the value of $d f l d t$.

[31] We fit median values of the sweep rate of the measured risers with the theoretical model (equation (9)) using a least squares method. In Figure 6 each diamond symbol represents the median value of the plasma density (particles per cc) versus median sweep rate $(\mathrm{kHz} / \mathrm{s})$ for one of the thirteen processed chorus events. The error bars correspond to the percentiles: $P_{\frac{16}{100}}$ and $P_{\frac{84}{100}}$. Red dashed lines describe results of the numerical simulation [Nunn et al., 2005, 2009]. The green dashed-dotted line represents the theoretical scaling based on the BWO model of Trakhtengerts et al. [2004] from equation (9). The free parameter $a$ from equation (9) is estimated using a least squares method and its value is $a=23.0 \pm 2.8$.

[32] Figure 7 shows the same data as in Figure 6 but only for risers, and with different fits superimposed. Blue thin

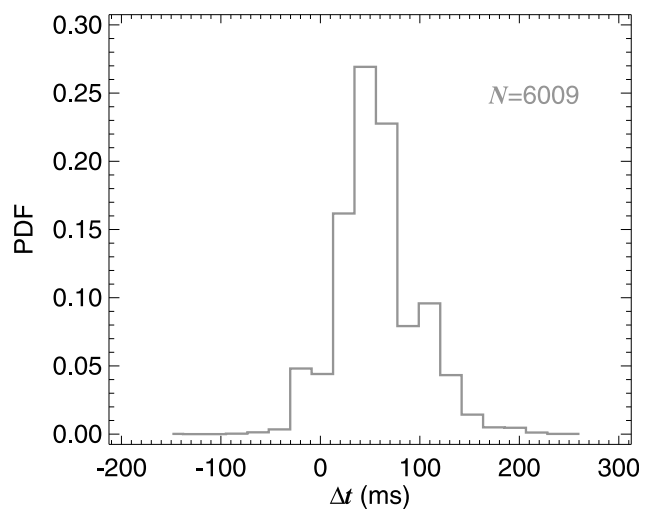

Figure 5. PDF of the duration of chorus elements measured on 6 December 2003. Negative values $\Delta t(\mathrm{~ms})$ represent falling tones with negative frequency sweep rates.

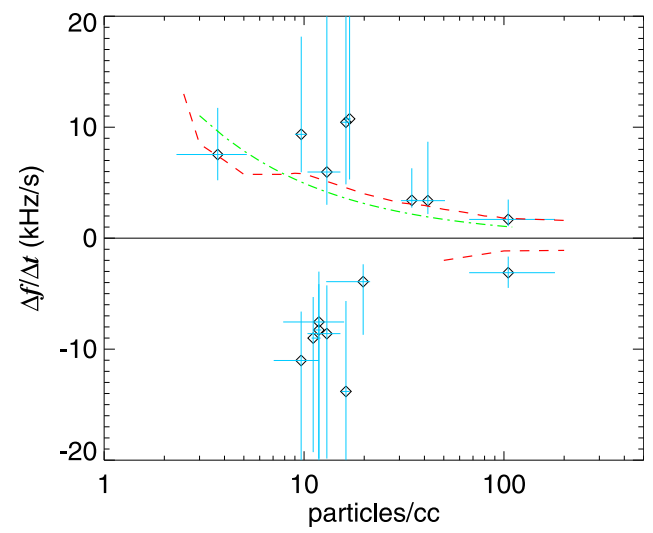

Figure 6. Each diamond symbol in the diagram characterizes the median value of the frequency sweep rate for one of the 13 time intervals of all chorus events (risers and fallers) versus the median value of the plasma density. The error bars describe percentiles $P_{\frac{16}{100}}$ and $P_{\frac{84}{100}}$. Red dashed lines represent results from the numerical simulation (risers are from Nunn et al. [2009] and fallers are from Nunn et al. [2005]), and the green dashed-dotted line represents theoretical scaling (see equation (9)) based on the BWO model of Trakhtengerts et al. [2004]. We fit median values of the sweep rate of the measured risers with the theoretical model (see equation (9)) using a least squares method.

lines are percentiles: $P_{\frac{16}{100}}$ and $P_{\frac{84}{100}}$. Red dashed lines shows results of the numerical simulation of risers from Nunn et al. [2009] and of fallers from Nunn et al. [2005]. The green dashed-dotted line describes the linear fit (logarithm of equation (11)) of theoretical scaling based on the model of Trakhtengerts et al. [2004] with the following input parameters: logarithm of the median plasma density, logarithm of the median value of $d f / d t$ for risers and measurement errors defined as $\left(\left(\log P_{\frac{16}{100}}-\log P_{\frac{84}{100}}\right) / 2\right)$. In this case the

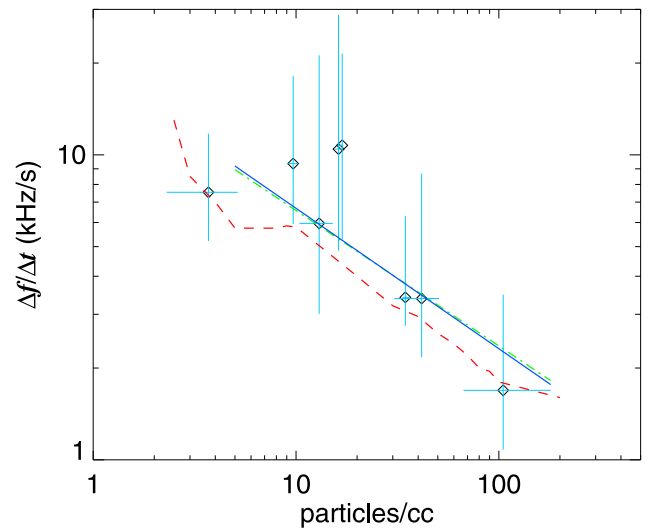

Figure 7. The same as in Figure 6 but on a log-log scale and with a theoretical scaling according to equation (11). The green dashed-dotted line describes a linear fit of the logarithm of the median of risers versus the logarithm of the median plasma density, taking into account measurement errors $\left(\log P_{\frac{16}{100}}-\log P_{\frac{84}{100}}\right) / 2$. The blue solid line shows a linear fit of the logarithm of the geometrical average of $P_{\frac{16}{10}}$ and $P_{\frac{84}{10}}$ versus the logarithm of the median plasma density, taking into account the same measurement errors. 
parameters from equation (11) are $a=18.3 \pm 1.8$ and $b=$ $-0.44 \pm 0.18$. The blue solid line is the linear fit with another set of input parameters: logarithm of the median plasma density, logarithm of the geometrical average of $P_{\frac{16}{100}}$ and $P_{\frac{84}{100}}$ and measurement errors defined as $\log \left(P_{\frac{84}{100}}^{\frac{100}{100}}\right.$ median value of risers). In this case the parameters from equation (11) are $a=19.3 \pm 1.7$ and $b=-0.46 \pm 0.17$. The value of the parameter $b$ is smaller than the theoretical scaling based on the BWO theory (equation (9)) but it is close to the theoretical value of $-2 / 3$ within the estimated experimental error.

\section{Discussion and Conclusions}

[33] We have investigated the frequency sweep rate of the chorus emissions as a function of the plasma density in the equatorial plane near $\mathrm{L}=4.2-4.6$. We calculate the plasma density from the value of the plasma frequency obtained by the WHISPER active sounder. The plasma frequency is sometimes well defined and values of the plasma frequency are accessible with a time resolution of a few seconds during the whole processed time interval. A limited number of values of the plasma frequency is available in other events. This is the reason why we used the median plasma densities for comparison with theoretical and simulation results. In some of the cases, there is a large uncertainty associated with the identification of the plasma frequency. For example, during 18 April 2002, intense natural waves are perturbing the identification of the plasma frequency in spectral signatures. Experimental errors are therefore equal to tens of percent of the median plasma density.

[34] The duration of individual chorus wave packets varied between 30 and $500 \mathrm{~ms}$. During the selected events, mostly risers occurred. Figures 6 and 7 show a general trend of increasing sweep rates with decreasing plasma density. This result was predicted by numerical simulations [Nunn et al., 2005, 2009] as well as by the theoretical scaling based on the BWO theory. It is noteworthy that the absolute values of $d f / d t$ for fallers show the same tendency as for risers; that is, they increase with decreasing plasma density.

[35] A more detailed comparison shows that the absolute values of the sweep rates of risers are in a good agreement with the numerical simulation within a density range of 20 105 particles per cubic centimeter. At the same time, the agreement is not so good for densities less than about 20 particles per cubic centimeter. For these low plasma densities the value of the frequency sweep rate is not uniquely determined. Measurements and numerical simulations give more than one value of the frequency sweep rate for one value of plasma density, because chorus wave packets have in this range of plasma densities random behavior and the value of the frequency sweep rate of individual wave packets vary very strongly. It is, however, possible, that the sweep rate of each element is a well determined function of the ambient plasma parameters: in some processed cases the plasma density fluctuations were rather large. In the area of high densities experimental results for fallers give slightly larger values of sweep rates than the numerical simulations. The comparison with the BWO theory shows a reasonable agreement of the general functional form (equation (9)) with the experimental data for risers. A more conclusive test would need more experimental data points and a better coverage of the given range of plasma densities, including the interval between 40 and 105 particles per cubic centimeter.

[36] The theoretical scaling based on the BWO theory is reproduced within statistical errors of the fit show in Figure 7. Following the discussion after equation (9), it is probable that the parameter $Q$ (defining excess of the energetic plasma density above the generation threshold) is not the same for all our cases. Therefore the parameter $a$ in equation (11) is not constant and this can bias the fit. However, the obtained rough agreement with the theory indicates that values of $Q$ are on average not substantially different for different $n_{e}$. On the other hand, we cannot exclude that $Q$ may vary strongly within the time interval of each event and cause the observed spread of $d f l d t$. To summarize, our study demonstrates that the plasma density is a parameter which is correlated with the characteristic frequency sweep rates of chorus elements. From the Table 1 is evident, that other factors (MLT, $L$ and $K_{p}$ ) were less significant for the value of $d f / d t$.

[37] As the theories of Trakhtengerts [1995, 1999], Trakhtengerts et al. [2004], and Omura et al. [2008, 2009] predict strong dependence of $d f / d t$ on the wave amplitude $B_{w}$, the work on experimental analysis of this effect is underway, and we will report our results when they become available.

[38] Acknowledgments. We thank the teams of the WBD, STAFF, and WHISPER instruments for the magnetic electric field data and plasma density. The WBD instrument is supported by NASA GSFC contract NNX07AI24G. The present work was supported under the GACR grant 205/10/2279, the ME 10001, LAPBIAT-2 Project RITA-CT-2006025969, the grant RFBR-CNRS N10-02-9311, by the Russian Foundation for Basic Research (grant 08-02-00967), and the General Physics Division of the Russian Academy of Sciences (the Program "Plasma Processes in Solar System").

[39] Robert Lysak thanks Mark Golkowski and another reviewer for their assistance in evaluating this paper.

\section{References}

Anderson, R. R., and K. Maeda (1977), VLF emissions associated with enhanced magnetospheric electrons, J. Geophys. Res., 84, 135-146.

Bell, T. F., U. S. Inan, N. Haque, and J. S. Pickett (2009), Source regions of banded chorus, Geophys. Res. Lett., 36, L11101, doi:10.1029/ 2009GL037629.

Bespalov, A. A., and A. G. Demekhov (2009), A linear theory of the backward-wave-oscillator regime in the magnetospheric cyclotron ELF/ VLF maser, Radiophys. Quantum Electron. (Engl. Trans1.), 52(11), 761-773

Burtis, W. J., and R. A. Helliwell (1969), Banded chorus: A new type of VLF radiation observed in the magnetosphere by OGO 1 and OGO 3, J. Geophys. Res., 74, 3002-3010.

Cornilleau-Wehrlin, N., et al. (1997), The Cluster spatio-temporal analysis of field fluctuations STAFF experiment, Space Sci., 79, 107-136.

Décréau, P. M. E., et al. (2001), Early results from the Whisper instrument on Cluster: An overview, Ann. Geophys., 19, 1241-1258.

Demekhov, A. G., V. Y. Trakhtengerts, M. J. Rycroft, and D. Nunn (2006), Electron acceleration in the magnetosphere by whistler-mode waves of varying frequency, Geomagn. Aeron. (Engl. Transl.), 46(6), 711-716.

Demekhov, J. G., and V. Y. Trakhtengerts (2008), Dynamics of the magnetospheric cyclotron ELF/VLF maser in the backward wave oscillator regime. II. The influence of the magnetic-field inhomogeneity, Radiophys. Quantum Electron. (Engl. Transl.), 51(11), 977-987.

Gołkowski, M., and U. S. Inan (2008), Multistation observation of ELF/ VLF whistler mode chorus, J. Geophys. Res., 113, A08210, doi:10.1029/2007JA012977.

Gurnett, D. A., and B. J. O'Brien (1964), High-latitude geophysical studies with satellite Injun 3, 5, very low frequency electromagnetic radiation, J. Geophys. Res., 69, 65-89.

Gurnett, D. A., W. S. Kurth, and F. L. Scarf (1981), Plasma waves near Saturn: Initial results from Voyager 1, Science, 212, 235-239. 
Gurnett, D. A., et al. (2001), First results from the Cluster wideband plasma wave investigation, Ann. Geophys., 19, 1259-1272.

Horne, R. B., and R. M. Thorne (1998), Potential waves for relativistic electron scattering and stochastic acceleration during magnetic storms, Geophys. Res. Lett., 25, 3011-3014, doi:10.1029/98GL01002.

Horne, R. B., and R. M. Thorne (2003), Relativistic electron acceleration and precipitation during resonant interactions with whistler-mode chorus, Geophys. Res. Lett., 30(10), 1527, doi:10.1029/2003GL016973.

Horne, R. B., S. A. Glauert, and R. M. Thorne (2003), Resonant diffusion of radiation belt electrons by whistler-mode chorus, Geophys. Res. Lett., 30(9), 1493, doi:10.1029/2003GL016963.

Horne, R. B., et al. (2005), Wave acceleration of electrons in the Van Allen radiation belts, Nature, 437, 227-230.

Hospodarsky, G. B., T. F. Averkamp, W. S. Kurth, D. A. Gurnett, J. D. Menietti, O. Santolik, and M. K. Dougherty (2008), Observations of chorus at Saturn using the Cassini radio and plasma wave science instrument, J. Geophys. Res., 113, A12206, doi:10.1029/2008JA013237.

Kennel, C. F., and H. E. Petschek (1966), Limit on stable trapped particle fluxes, J. Geophys. Res., 71, 1-28.

LeDocq, M. J., D. A. Gurnett, and G. B. Hospodarsky (1998), Chorus source locations from VLF Poynting flux measurements with the Polar spacecraft, Geophys. Res. Lett., 25, 4063-4066.

Li, W., et al. (2010), THEMIS analysis of observed equatorial electron distributions responsible for chorus excitation, J. Geophys. Res., 115, A00F11, doi:10.1029/2009JA014845.

Manninen, J. (2005), Some aspects of ELF-VLF emissions in geophysical research, Publ. 98, Sodankylä Geophys. Obs. Publ., Sodankylä, Finland

Menietti, J. D., R. B. Horne, D. A. Gurnett, G. B. Hospodarsky, C. W. Piker, and J. B. Groene (2008), A survey of Galileo plasma wave instrument observations of Jovian whistler-mode chorus, Ann. Geophys., 7, 1819-1828.

Meredith, N. P., R. B. Horne, and R. R. Anderson (2001), Substorm dependence of chorus amplitudes: Implications for the acceleration of electrons to relativistic energies, J. Geophys. Res., 106, 13,165-13,178.

Meredith, N. P., R. B. Horne, R. H. A. Iles, R. M. Thorne, D. Heynderickx, and R. R. Anderson (2002), Outer zone relativistic electron acceleration associated with substorm-enhanced whistler mode chorus, J. Geophys. Res., 107(A7), 1144, doi:10.1029/2001JA900146.

Meredith, N. P., M. Cain, R. B. Horne, R. M. Thorne, D. Summers, and R. R. Anderson (2003), Evidence for chorus-driven electron acceleration to relativistic energies from a survey of geomagnetically disturbed periods, J. Geophys. Res., 108(A6), 1248, doi:10.1029/2002JA009764.

Nunn, D. (1990), The numerical simulation of VLF nonlinear wave-particle interactions in collision-free plasmas using the Vlasov hybrid simulation technique, Comput. Phys. Commun., 60, 1-25.

Nunn, D., Y. Omura, H. Matsumoto, I. Nagano, and S. Yagitani (1997), The numerical simulation of VLF chorus and discrete emissions observed on the Geotail satellite using a Vlasov code, J. Geophys. Res., 102, 27,083-27,097.

Nunn, D., M. Rycroft, and V. Trakhtengerts (2005), A parametric study of the numerical simulations of triggered VLF emissions, Ann. Geophys., $23,3655-3666$.

Nunn, D., O. Santolik, M. Rycroft, and V. Trakhtengerts (2009), On the numerical modelling of VLF chorus dynamical spectra, Ann. Geophys. 27, 2341-2359.

Omura, Y., Y. Katoh, and D. Summers (2008), Theory and simulation of the generation of whistler-mode chorus, J. Geophys. Res., 113, A04223, doi:10.1029/2007JA012622.

Omura, Y., M. Hikishima, Y. Katoh, D. Summers, and S. Yagitani (2009), Nonlinear mechanisms of lower-band and upper-band VLF chorus emissions in the magnetosphere, J. Geophys. Res., 114, A07217, doi:10.1029/ 2009JA014206.
Santolík, O., M. Parrot, and F. Lefeuvre (2003), Singuar value decomposition methods for wave propagation analysis, Radio Sci., 38(1), 1010, doi:10.1029/2000RS002523.

Santolík, O., D. A. Gurnett, J. S. Pickett, M. Parrot, and N. CornilleauWehrlin (2004a), A microscopic and nanoscopic view of storm-time chorus on 31 March 2001, Geophys. Res. Lett., 31, L02801, doi:10.1029/ 2003GL018757.

Santolík, O., D. A. Gurnett, and J. S. Pickett (2004b), Multipoint investigation of the source region of storm-time chorus, Ann. Geophys., 22, $2555-2563$.

Santolík, O., E. Macusova, E. Titova, B. Kozelov, D. Gurnett, J. Picket, V. Trakhtengerts, and A. Demekhov (2008), Frequencies of wave packets of whistler-mode chorus inside its source region: A case study, Ann. Geophys., 26, 1665-1670.

Sazhin, S. S., and M. Hayakawa (1992), Magnetospheric chorus emissions: A review, Planet. Space Sci., 40, 681-697.

Storey, L. R. O. (1953), An investigation of whistling atmospherics, Philos Trans. R. Soc. London A, 246, 113-141.

Trakhengerts, V. I. (1963), On generation mechanism of VLF emissions in external Earth's radiation belts, Geomagn. Aeron. (Engl. Transl.), 3(3), $442-451$.

Trakhtengerts, V. Y. (1995), Magnetosphere cyklotron maser: Backward wave oscillator generation regime, J. Geophys. Res., 100, 205-210.

Trakhtengerts, V. Y. (1999), A generation mechanism for chorus emission, Ann. Geophys., 17, 95-100.

Trakhtengerts, V. Y., A. G. Demekhov, E. E. Titova, B. V. Kozelov, O. Santolik, D. Gurnett, and M. Parrot (2004), Interpretation of cluster data on chorus emissions using the backward wave oscillator model, Phys. Plasmas, 11, 1345-1351.

Trakhtengerts, V. Y., et al. (2007), Formation of VLF chorus frequency spectrum: Cluster data and comparison with the backward wave oscillato model, Geophys. Res. Lett., 34, L02104, doi:10.1029/2006GL027953.

Tsurutani, B. T., and E. J. Smith (1977), Two types of magnetospheric ELF chorus and their substorm dependences, J. Geophys. Res., 82, $5112-5128$.

Tsurutani, B. T., and E. M. Smith (1974), Postmidnight Chorus: A substorm phenomenon, J. Geophys. Res., 79, 118-127.

P. Décréau, J.-L. Rauch, and J.-G. Trotignon, Laboratoire de Physique et Chimie de l'Environnement et de l'Espace, CNRS, University of Orléans, 3A, Ave. de la Recherche Scientifique, F-45071 Orléans, Cédex 2, France. (pdecreau@cnrs-orleans.fr)

A. G. Demekhov, Institute of Applied Physics, Russian Academy of Sciences, 46 Ul'yanova Str., Nizhny Novgorod 603950, Russia. (andrei@ appl.sci-nnov.ru)

D. A. Gurnett and J. S. Pickett, Department of Physics and Astronomy, University of Iowa, Iowa City, IA 52242-1479, USA. (donald-gurnett@ uiowa.edu; pickett@uiowa.edu)

B. V. Kozelov and E. E. Titova, Polar Geophysical Institute, 14 Fersmana Str., Apatity 184209, Russia. (lena.titova@gmail.com; boris.kozelov@ gmail.com)

E. Macúšová, Faculty of Mathematics and Physics, Charles University, V Holešovičkách 2, 18 000, Prague, Czech Republic. (eva.macusova@ mff.cuni.cz)

D. Nunn, ECS, Southampton University, Southampton SO17 1BJ, UK (dn@ecs.soton.ac.uk)

O. Santolík, Institute of Atmospheric Physics, Academy of Sciences of the Czech Republic, Boční II 1401, 14131 Prague 4, Czech Republic. (ondrej.santolik@mff.cuni.cz) 